\title{
Universiteit
}

Leiden

The Netherlands

\section{Design and performance of a high-resolution frictional force microscope with quantitative three-dimensional force sensitivity}

Dienwiebel, M.; Kuyper, E. de; Crama, L.; Frenken, J.W.M.; Heimberg, J.A.; Spaanderman, D.J.; ... ; Glastra, van Loon D.

\section{Citation}

Dienwiebel, M., Kuyper, E. de, Crama, L., Frenken, J. W. M., Heimberg, J. A., Spaanderman, D. J., ... Drift, E. van der. (2005). Design and performance of a high-resolution frictional force microscope with quantitative three-dimensional force sensitivity. Review Of Scientific Instruments, 76, 043704. doi:10.1063/1.1889233

Version: $\quad$ Not Applicable (or Unknown)

License: $\quad$ Leiden University Non-exclusive license

Downloaded from: https://hdl.handle.net/1887/61358

Note: To cite this publication please use the final published version (if applicable). 


\section{Design and performance of a high-resolution frictional force microscope with quantitative three-dimensional force sensitivity}

M. Dienwiebel, E. de Kuyper, L. Crama, J. W. M. Frenken, J. A. Heimberg, D.-J. Spaanderman, D. Glastra van Loon, T. Zijlstra, and E. van der Drift

Citation: Review of Scientific Instruments 76, 043704 (2005); doi: 10.1063/1.1889233

View online: http://dx.doi.org/10.1063/1.1889233

View Table of Contents: http://aip.scitation.org/toc/rsi/76/4

Published by the American Institute of Physics

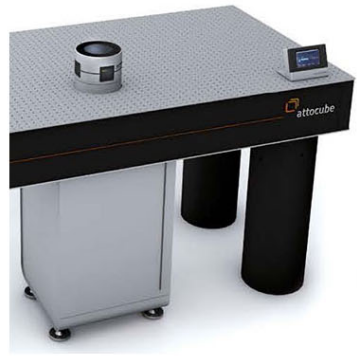

Obstruction free access 


\title{
Design and performance of a high-resolution frictional force microscope with quantitative three-dimensional force sensitivity
}

\author{
M. Dienwiebel, ${ }^{\text {a) }}$ E. de Kuyper, L. Crama, and J. W. M. Frenken ${ }^{\text {b) }}$ \\ Kamerlingh Onnes Laboratory, Leiden University, P.O. Box 9506, 2300 RA Leiden, The Netherlands \\ J. A. Heimberg, ${ }^{\text {() }}$ D.-J. Spaanderman, and D. Glastra van Loon \\ FOM-Institute for Atomic and Molecular Physics, Kruislaan 407, 1098 SJ Amsterdam, The Netherlands \\ T. Zijlstra and E. van der Drift \\ Delft Institute of Microelectronics and Submicron Technology (DIMES), Delft University of Technology, \\ P.O. Box 5053, 2600 GB Delft, The Netherlands
}

(Received 17 March 2004; accepted 22 January 2005; published online 1 April 2005)

\begin{abstract}
In this article, the construction and initial tests of a frictional force microscope are described. The instrument makes use of a microfabricated cantilever that allows one to independently measure the lateral forces in $\mathrm{X}$ and $\mathrm{Y}$ directions as well as the normal force. We use four fiber-optic interferometers to detect the motion of the sensor in three dimensions. The properties of our cantilevers allow easy and accurate normal and lateral force calibration, making it possible to measure the lateral force on a fully quantitative basis. First experiments on highly oriented pyrolytic graphite demonstrate that the microscope is capable of measuring lateral forces with a resolution down to 15 pN. () 2005 American Institute of Physics. [DOI: 10.1063/1.1889233]
\end{abstract}

\section{INTRODUCTION}

One of the oldest unresolved problems in physics concerns mechanisms of friction. This may seem surprising in the light of the fact that systematic research dates back to Leonardo da Vinci. ${ }^{1}$ There is an impressive body of phenomenological knowledge on friction, but most of this knowledge lacks a true understanding on a microscopic level. Questions about atomic-scale details of energy dissipation are becoming increasingly important as, e.g., technologies of data storage, microelectromechanical systems (MEMS) and specialized coatings advance. In these areas, the phenomenological and often used friction law of Amontons and Coulomb, that the frictional force is linearly proportional to the normal force, does not always apply., ${ }^{2,3}$ In order to better predict and control tribological behavior on the small length scales involved, a truly microscopic understanding of friction and wear is required. Real surfaces in contact only truly meet at those points with the highest topography due to surface roughness (i.e., asperities). It has been shown that it is the statistics of these asperities that leads to the Amontons and Coulomb law. ${ }^{4}$ As asperities move with respect to each other, the contributions of individual asperities to the friction force are averaged. One approach to obtain information on the tribological behavior of single asperities is to control the surfaces such that only one asperity is created. This area of research has come to be called nanotribology. It has long been recognized that the tip-on-flat geometry of an atomic

\footnotetext{
${ }^{a)}$ Present address: IAVF Antriebstechnick AG, Im Schlehert 32, 76187 Karlsruhe, Germany.

${ }^{\text {b) }}$ Electronic mail: frenken@phys.leidenuniv.nl

${ }^{c}$ Present address: John Hopkins Applied Physics Laboratory, 11100 John Hopkins Rd., Laurel, Maryland 20723-6099.
}

force microscope (AFM) closely resembles a realistic asperity. ${ }^{5}$ AFMs have been adapted to measure lateral forces down to the nanometer and nanonewton regimes by measuring the torsional response of the force probe. In the past, frictional force microscopes (FFMs) have produced predominantly qualitative results although recently some groups have succeeded in obtaining quantitative nanotribology results. ${ }^{3,6}$ Even as the techniques in traditional FFM become more refined, the basic problem remains that this method uses a force probe designed to be most sensitive to forces normal to the contact. The ramification of this is that most cantilevers snap into contact as the tip-to-sample distance approaches the near contact regime, so that the lateral forces are measured in "hard" contact. This near-contact regime is of great technological importance as fly heights in disk drives decrease and as the length scales in MEMS are reduced. Cantilevers that do not suffer from the "snap-to-contact" problem will have large torsional spring constants so that small lateral forces cannot be detected. In most FFMs, only one component of the lateral force is measured. In addition, bending motion of standard cantilevers cannot be distinguished from buckling ${ }^{7}$ and it is very difficult to minimize coupling between the normal and the torsional bending, ${ }^{8}$ which can be seen by the fact that the feedback parameters can have dramatic influence on the measured friction signal. ${ }^{9}$ This is also a fact well known and of equal frustration to the AFM community. Our main objective was to build a quantitative FFM that would measure both lateral forces with better sensitivity and at smaller tip-sample separation distances than current FFMs. Ultimately it is our aim to atomically control the contact area between the tip and sample in UHV via field ion microscopy and field evaporation. ${ }^{10}$ Consideration of these requirements and future additions played an important role in 

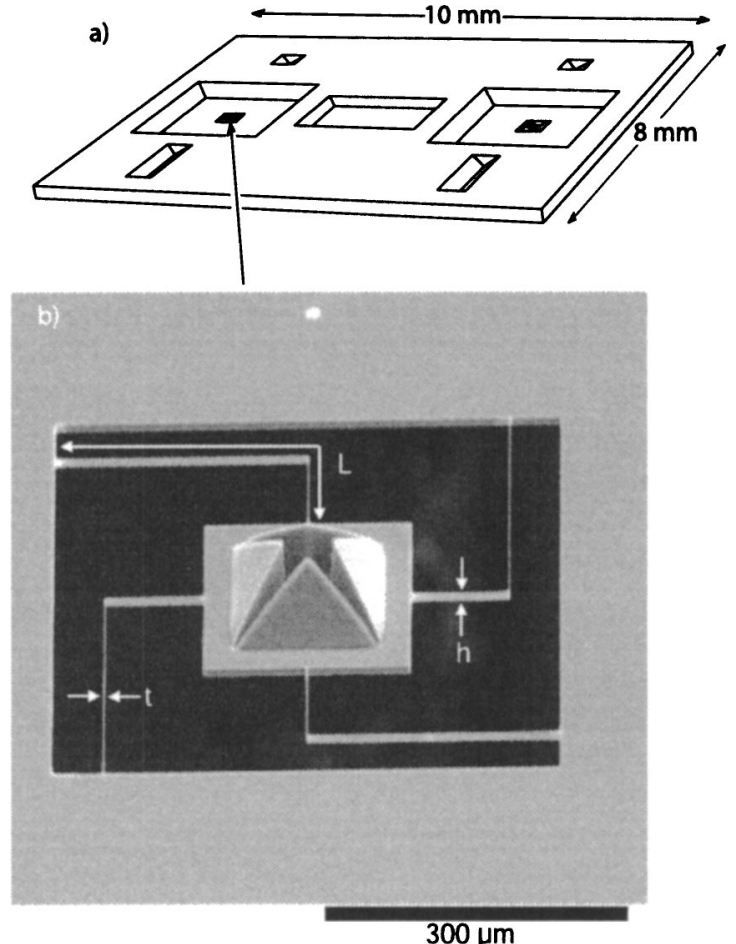

FIG. 1. Schematic of the "Tribolever®" device (a). The prototype chip $(10 \mathrm{~mm} \times 8 \mathrm{~mm})$ includes two force sensors, each with its own set of kinematic mounts. SEM micrograph (b) of the sensor. The dimensions of the legs of the sensor are $L=350 \mu \mathrm{m}, t=1.4 \mu \mathrm{m}$ and $h=10.6 \mu \mathrm{m}$.

some of the design decisions for our prototype, ambientcondition FFM with three-dimensional detection. The instrument consists of two stages: the fiber head assembly containing the detection system and the specialized lateral force cantilever, and the sample stage that allows for manipulation of the sample with respect to the tip and cantilever. We began our FFM design by first designing a dedicated force probe. The precise design and manufacturing details of this socalled "Tribolever®" have been reported in another article. ${ }^{11}$ A scanning electron microscope (SEM) image of the monocrystalline monolithic silicon structure is seen in Fig. 1. Four high aspect ratio legs extend out from a central detection pyramid. A fiber optic interferometer reflects off of each pyramid face to track the motion of the pyramid. The scanning tip, which can be an etched metal wire, for example, tungsten, is threaded through the central hole of the pyramid and extends $\sim 50-100 \mu \mathrm{m}$ out from the base of the pyramid to interact with the surface. The shape and dimensions of the four legs have been chosen and tested using finite element analysis, ${ }^{12}$ so that the two lateral spring constants are equal and significantly lower than the torsional spring constant of single board-type cantilevers used in AFMs. Using cantilever dimensions obtained from the literature, ${ }^{13}$ a typical torsional spring constant is $72 \mathrm{~N} / \mathrm{m}$, whereas the Tribolevers $®$ lateral spring constants are typically $k_{x}^{\text {Tribolever }} \equiv k_{y}^{\text {Tribolever }}=1.4 \mathrm{~N} / \mathrm{m}$. In the normal direction, $k_{z}^{\mathrm{AFM}}=0.2 \mathrm{~N} / \mathrm{m}$, while $k_{z}^{\text {Tribolever }}$ $=10.6 \mathrm{~N} / \mathrm{m}$ (see below). Additionally, the coupling in the Tribolever between the three orthogonal directions is $\sim 10^{-6}$ and coupling between torsional (out of scanning plane) and normal forces is essentially zero. The focus of the present article is on the apparatus, which houses, moves and manipu- lates the optical fibers with respect to the detection pyramid and the tip-fiber head with respect to the sample. Initial results are reported, and used to demonstrate the performance of this microscope.

\section{INSTRUMENTATION}

\section{A. Detection principle}

As discussed briefly above, the pyramid is in the center of the detection system, which consists of four all-fiber interferometers. Each of the four glass fibers is coupled to a laser diode and a photodiode detector as described in Sec. II B. The light that leaves the fiber at the end face is centered on one of the four pyramid faces. Part of that light is reflected back into the fiber, where it interferes with the light that is reflected internally at the fiber end face. The interferometer's output is given by

$$
I=I_{0}\left[1+V \cos \left(2 \pi \frac{2 D}{\lambda}\right)\right],
$$

where $I$ is the output current, $V$ is the relative interference amplitude, $D$ is the fiber-sample distance, and $\lambda$ is the wavelength of the laser (780 nm in our case). The offset $I_{0}$ and the amplitude $V$ are both determined by the reflectivities of the two interfaces responsible for the interference signal. Specifically, for the fiber/air interface a maximum reflectance of $4 \%$ is expected and for the pyramid surface a maximum reflectance in the order of $60 \%$. The pyramid, which is approximately $80 \mu \mathrm{m}$ high, with a $150-\mu \mathrm{m}$-wide base, is formed via a $\mathrm{KOH}$ wet etch, which exposes the (111) planes of silicon, which provides an area available for the fibers to reflect from of at least $1500 \mu \mathrm{m}^{2}$. A special passivation technique, necessary to protect the corners of the convex structure from underetching, ${ }^{11}$ gives rise to the presence of a cross-shaped hole within the pyramid (Fig. 1). One advantage of this design is that the entire cantilever structure can be made out a single-crystal silicon wafer. The faces of the pyramid are highly reflective and of a well-defined angle, $\theta$ $=54.74^{\circ}$, with respect to the (100) surface plane of the wafer. If each of the four glass fibers is adjusted such that the light intensity increases when the fiber-pyramid distance decreases [Eq. (1)] the three-dimensional displacement with respect to the fixed fibers can be extracted from the normalized sum and differences of the signals coming from the four interferometers. ${ }^{14}$ These linear combinations need to be weighted by the appropriate geometrical projection (Fig. 2)

$$
\begin{aligned}
& X=\frac{X_{2}-X_{1}}{2 \sin \theta}, \\
& Y=\frac{Y_{2}-Y_{1}}{2 \sin \theta}, \\
& Z=\frac{X_{1}+X_{2}+Y_{1}+Y_{2}}{4 \cos \theta} .
\end{aligned}
$$

\section{B. Interferometers}

The design of our interferometers follows closely those discussed in the literature, ${ }^{15,16}$ with attention given to the 


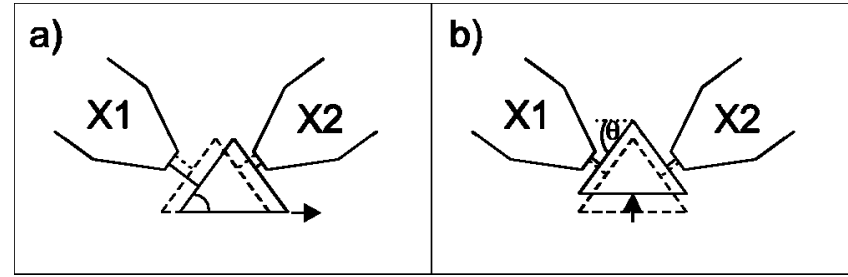

FIG. 2. Schematic showing two of the four glass fibers. If the pyramid moves laterally (a), the distance X1 between the left glass fiber and the pyramid increases and the distance $\mathrm{X} 2$ between the right glass fiber and the pyramid decreases or vice versa. If the pyramid moves normal to the sample surface, both distances either decrease or increase. This allows one to extract the displacements of the pyramid in the $\mathrm{X}$ and $\mathrm{Z}$ directions. Similarly, from the other fiber pair one obtains the displacements in the $\mathrm{Y}$ and $\mathrm{Z}$ directions. The displacements are extracted from the distance changes, according to Eqs. (2)-(4).

stability of the laser diode's output intensity and wavelength. ${ }^{17}$ One unique aspect of our design is that each opposing pair of interferometers is driven by a single laser diode so that the influence of fluctuations in laser intensity and wavelength is greatly reduced, while the remaining variations can be divided out by use of a reference signal. A schematic of one such pair (e.g., the X pair) is seen in Fig. 3.

Light coming from the laser diode is first divided over two branches using a bidirectional $2 \times 2$ fiber coupler. The two branches are denoted with $\mathrm{X} 1$ and $\mathrm{X} 2$, respectively. A second $2 \times 2$ coupler in each arm completes the interferometer, by coupling out the backwards traveling reflected light into the photodiode detector. The same coupler couples out

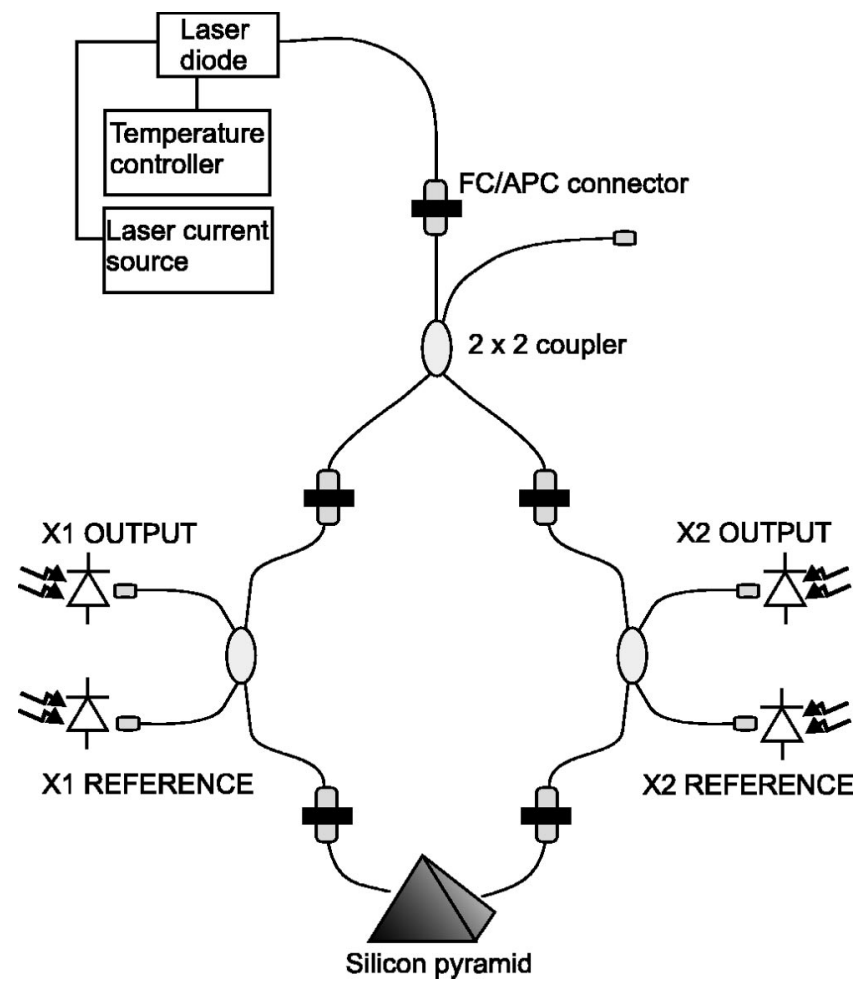

FIG. 3. Components of one interferometer pair. The interferometer can be divided into three distinct sections: the laser system, the fiber system and the detection system. The laser system consists of the laser diode with integrated Faraday isolator and the controlling power supplies. The fiber system consists of couplers, connectors, adapters and the fiber itself. The detection system consists of photodiodes and supporting electronics.

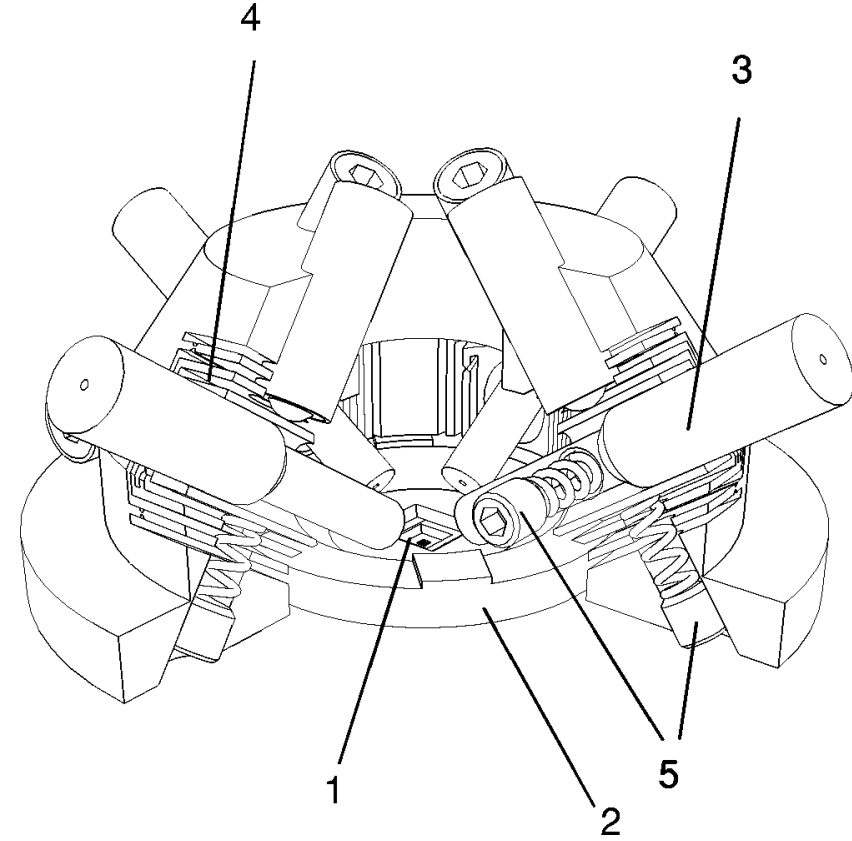

FIG. 4. Schematic drawing of the fiber positioning head cut open for illustration. (1) Tribolever®, (2) Tribolever® support plate, (3) Nanomotor®, (4) flexure hinge, and (5) adjustment screws.

$50 \%$ of the the primary light into the reference signal detector.

We see no evidence for optical coupling between the two fiber pairs. Such coupling was not expected, as little diffuse reflection occurs at the pyramid faces. As a result, we see no change in one pair, even when the other pair is optically disconnected. In order for the $125-\mu \mathrm{m}$-diam fibers to be positioned close the pyramid faces, they must be tapered to a maximum end face diameter of $80 \mu \mathrm{m} .{ }^{18}$ We have used both sharpened fibers with cleaved end faces ${ }^{19}$ and fibers chemically etched using the liquid layer protection procedure. ${ }^{20}$ The latter method results in fiber tips with a cone angle that can be varied from $8^{\circ}$ to $41^{\circ}$ depending on the protection fluid. ${ }^{21}$ To create an end face the fiber tips were mechanically polished. The former method results in slightly stronger interference signals (presumably due to the cleave). The latter method routinely produces end face diameters on the order of $30 \mu \mathrm{m}$, which allows for more flexibility when positioning all four fibers.

\section{Fiberhead}

To position the four fibers with respect to the silicon pyramid we constructed a special fiberhead (see Fig. 4). The fiberhead was machined by spark erosion from a single block of low-thermal-expansion metal (Invar). The distance of the end face of each fiber with respect to the pyramid face is adjusted by miniature inertial piezomotors (Nanomotors ${ }^{\circledR 2}{ }^{22}$ ), that can either be driven in discrete steps over a maximum distance of approximately $4 \mathrm{~mm}$ or be adjusted continuously with sub- $\AA$ resolution over a range of $400 \mathrm{~nm}$. The first mode allows one to retract the glass fibers to a safe distance during change of sensors, the latter is used to calibrate the interferometer signals and to position the fibers at the distance of maximum sensitivity. Additionally the continuous 


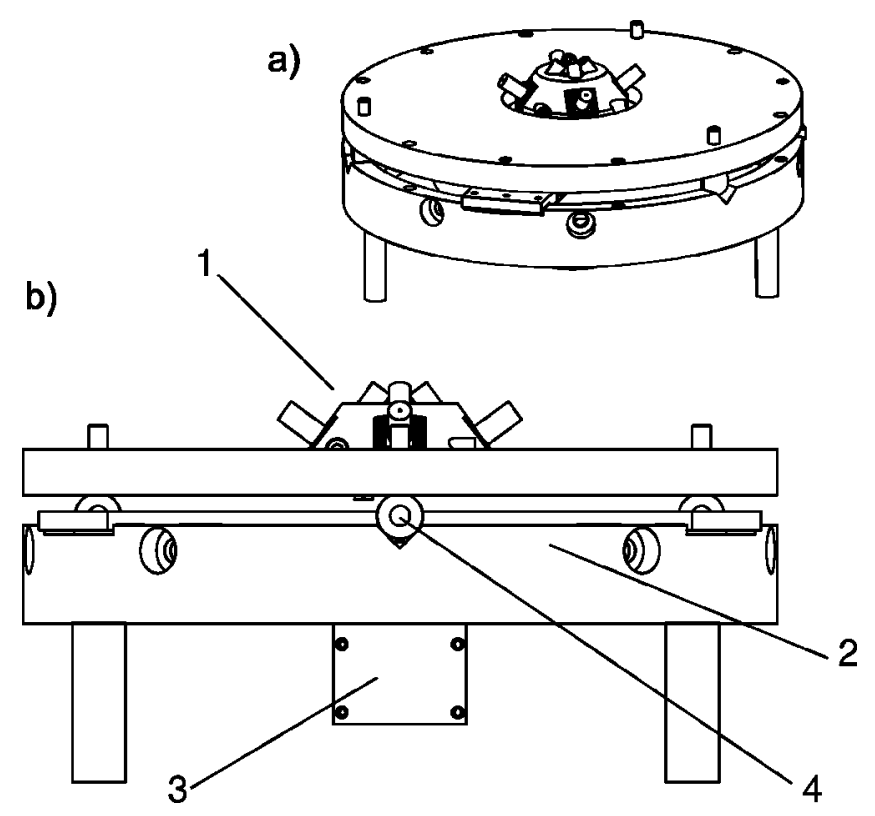

FIG. 5. (a) Perspective drawing of the microscope assembly. (b) Side view with (1) fiber positioning head, (2) XY motor, (3) Z coarse approach motor, (4) kinematic mount between the motor/sample stage and the fiber positioning head.

mode can be used to compensate possible drift between the fibers and the pyramid due to thermal expansion of the microscope (see electronics section). The Nanomotors ${ }^{\circledR}$ are mounted in miniature flexure hinge springs, which are part of the fiber head. These springs allow the adjustment of each fiber axis in a plane parallel to the pyramid plane.

Different types of the Tribolever ${ }^{\circledR}$ can be clamped onto an exchangeable plate by means of two stiff leaf springs. On the backside of the cantilever plate, three small diameter ruby spheres are cemented. A quadrahedral hole-groove-flattype kinematic mount is integrated into the silicon chip that houses the Tribolever®. ${ }^{23}$ The ruby spheres rest within the kinematic mount on each silicon chip. We have found that this system of kinematic mounts works so well that only little repositioning $(<10 \mu \mathrm{m})$ of the fibers via the flexure hinge springs is necessary between different cantilevers to restore optimal signals.

The fiberhead is fixed into an invar plate that matches the dimensions of the sample stage underneath. The complete assembly of the microscope can be seen in Fig. 5. The sample (restricted to lateral dimensions of $10 \times 10 \mathrm{~mm}^{2}$ ) sits on a scan tube ${ }^{24}$ which rests inside a set of nested inertial piezo motors that allow for four-dimensional motion of the sample with respect to the tip: $\mathrm{X}, \mathrm{Y}, \mathrm{Z}$ and rotation $(\Phi)$. The $\mathrm{Z}$ and $\mathrm{X}-\mathrm{Y}$ motors are similar to those discussed by Hug et $a l .{ }^{25}$ The scanner is directly coupled to the $\mathrm{Z}$ coarse approach motor. The $\mathrm{Z}$ motor is located in the center of an X-Y- $\Phi$ motor, which allows for long range manipulation of sample with respect to the tip. The rotation can be used to rotate the lattice planes of a blunt tip and the sample with respect to each other to measure variations in friction forces that are introduced when the tip and sample lattices are brought in and out of registry. ${ }^{26}$ The X-Y- $\Phi$ motor consists of a sapphire disk of $100 \mathrm{~mm}$ diameter, which is clamped between three pairs of piezo stacks using $\mathrm{CuBe}$ leaf springs. The rotational

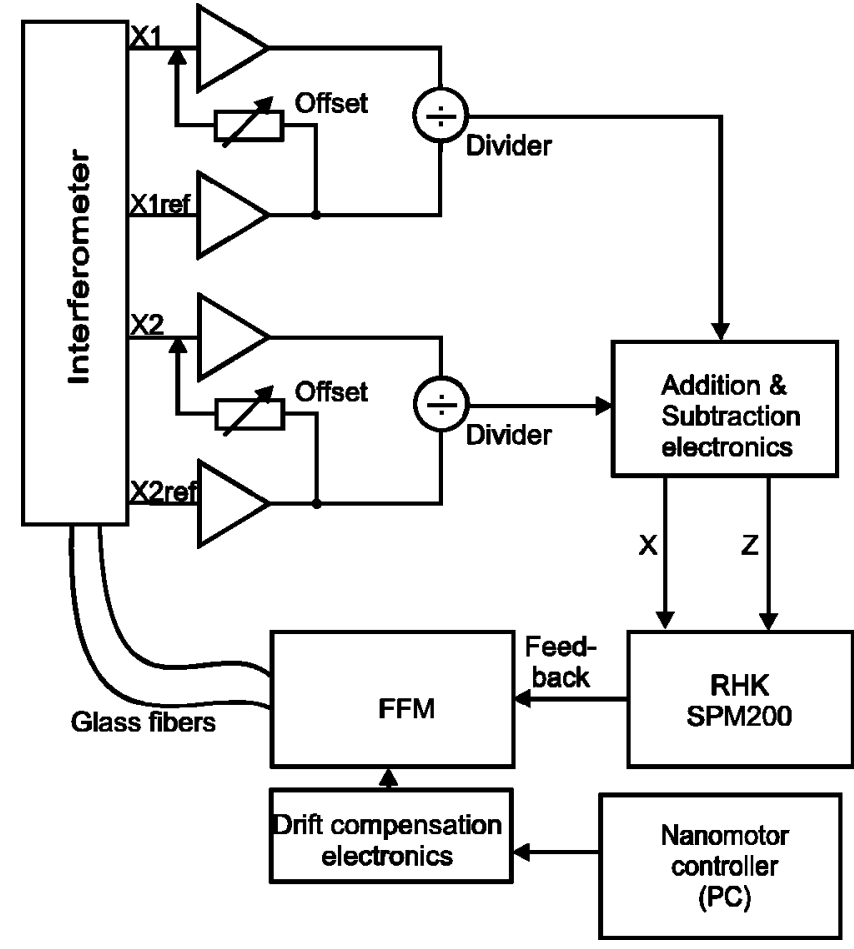

FIG. 6. Block diagram of the microscope's electronics (shown again only for the $\mathrm{X}$ pair). From the $\mathrm{X} 1$ and $\mathrm{X} 2$ signals coming from the interferometer an adjustable fraction of the reference signal is subtracted before the first amplification. The result is divided by the reference signal. This procedure allows the maximum amplification of the signal while introducing the lowest noise level. In the addition and subtraction electronics, the outputs from the X1 and X2 dividers are combined according to Eqs. (2)-(4) in order to obtain voltages that correspond to the true displacement of the Tribolever (X and $\mathrm{Z}$ ). These voltages are then fed into a commercial scan electronics system, which acquires the measured data and controls the sample motion (scanning and feedback).

motion of the X-Y- $\Phi$ motor is achieved by three shear piezos, which are arranged $120^{\circ}$ rotated with respect to each other. On each of these piezos a stack of shear piezos is glued for the X-Y motion.

\section{Electronics}

The system electronics can be split into two main components: data acquisition and sample motion (see Fig. 6).

As discussed in Sec. II A, the signal coming from each interferometer consists of a sinusoidal interference component plus an offset, which is due to the difference in reflection amplitudes. In our detection electronics, we first subtract a fraction of the reference signal and amplify only the interference component. The amplified signal is then divided by the reference signal to reduce the effect of fluctuations in laser diode intensity. The four resulting signals are then added and subtracted according to Eq. (4) to produce the three-dimensional Tribolever displacement information. All signals are then used as input for an RHK SPM200 system with added input capabilities so that the fully threedimensional motion of the tip can be monitored in real time.

A common problem in force microscope setups using fiber optic interferometry is drift between the fiber end face and the cantilever due to thermal expansion of the microscope. ${ }^{27}$ Although we have used materials with low 
a)
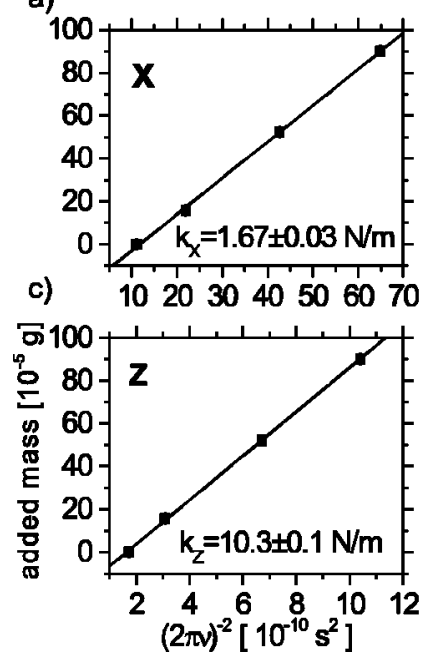

b)
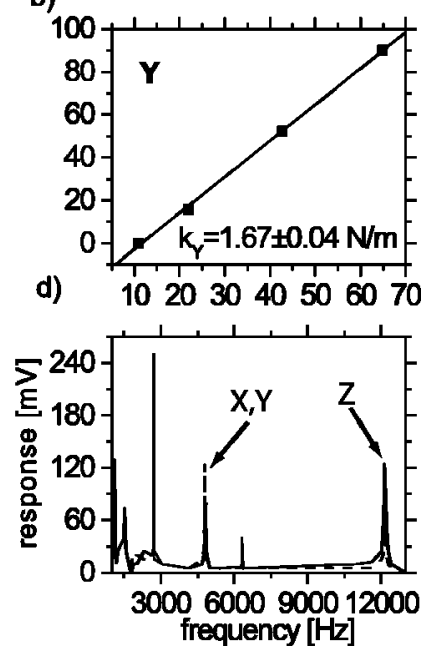

FIG. 7. Calibration data for one Tribolever® using the added mass method. Plots of the added mass vs frequency of the resonance peak (a) for the $X$ direction, (b) for Y direction, and (c) for Z direction. (d) Typical resonance spectrum of the microscope and the Tribolever® without added mass in the $\mathrm{X}$ direction (solid line) and in the $\mathrm{Y}$ direction (dashed line).

thermal expansion coefficients for the fiberhead components, the fiber-pyramid distance drifts slowly due to temperature variations in our nonclimatized laboratory. Using home-built electronics we apply very slow voltage ramps to the Nanomotors ${ }^{\circledR}$, to keep the fiber-pyramid distance constant for several hours.

\section{E. Calibration}

One of the extreme advantages of the Tribolever® is that it allows easy, yet very precise calibration. We routinely calibrate each Tribolever® prior to its first use. By exciting the Tribolever ${ }^{\circledR}$ acoustically with a loudspeaker, that is placed close to the fiberhead, frequencies of the resonances in the $\mathrm{X}$, $\mathrm{Y}$ and $\mathrm{Z}$ directions can be measured. Soda lime glass beads ${ }^{28}$ with masses ranging from 1.57 to $9.01 \mu \mathrm{g}$ have been placed on the central cross of the pyramid. By measuring the resonance frequencies as a function of the added mass, extremely accurate values of the Tribolever's ${ }^{\circledR}$ lateral and vertical spring constants have been determined. ${ }^{29}$ Figure 7 is an example of one such calibration run. This calibration procedure has no effect on the Tribolever® because the sphere is held in place by gravity. Calibration of the lateral (torsional) spring constant on traditional AFM cantilevers is more time consuming, more complex and significantly less accurate. ${ }^{30}$

Measured lateral spring constants in this example (Fig. 7) are $k_{X}=(1.67 \pm 0.03) \mathrm{N} / \mathrm{m}$ and $k_{Y}=(1.67 \pm 0.04) \mathrm{N} / \mathrm{m}$. These two spring constants are virtually identical and they are close to the value of $1.4 \mathrm{~N} / \mathrm{m}$ calculated from the dimensions of the legs of the Tribolever ${ }^{\circledR}$ using finite element analysis. The measured vertical spring constant for the Tribolever® is $k_{Z}^{\text {Tribolever }}=(10.3 \pm 0.1) \mathrm{N} / \mathrm{m}$ as compared to the calculated value of $25.8 \mathrm{~N} / \mathrm{m}$. The large deviation is due to the additional flexibility of a thin diaphragm $(2 \mathrm{~mm}$ $\times 2 \mathrm{~mm} \times 10.6 \mu \mathrm{m})$ that supports the Tribolever® on the silicon chip. This diaphragm is the result of a wet etch step

that forms a wide, recessed window to allow room for the detection fiber's access to the pyramid (Fig. 1).

In the design of the Tribolever $®$ device the geometry of window was changed to overcome this problem.

We also used the resonance spectra of the Tribolever® to estimate the noise levels of the optical detection and the electronics. With a spectrum analyzer, we measured the thermally excited $\mathrm{X}$ and $\mathrm{Y}$ resonances of a Tribolever® with lateral spring constants of $5.75 \mathrm{~N} / \mathrm{m}$. The amplitude of the resonant motion can be calculated by the equipartition theorem $\frac{1}{2} k_{x} x_{\mathrm{rms}}^{2}=\frac{1}{2} k_{B} T$, where $x_{\mathrm{rms}}$ is the root mean square thermal motion amplitude, $k_{B}$ is the Boltzmann constant and $T$ is the temperature. If the electronic instrument noise is much smaller than the thermal motion of the sensor, the root mean square voltage noise $V_{\text {rms }}$ at the resonance frequency is given by the relation $\alpha V_{\text {rms }}=x_{\text {rms }}=\sqrt{k_{B} T / k_{x}}{ }^{31} \alpha$ is a known calibration factor that relates the output voltage to the displacement of the Tribolever®. We compared the measured $V_{\text {rms }}$ with the calculated value of $V_{\mathrm{rms}}$ at the thermal limit. We found that the detected noise in the frequency range of the lateral resonances $(9.38 \mathrm{kHz})$ is a factor of $1.9(\mathrm{X} 1)-4.8$ (Y2) higher than the thermal noise. In a FFM measurement, the noise levels are certainly different. Typical signal frequencies are lower (below $2-3 \mathrm{kHz}$ ) and the tip is in contact with a surface. However, the measured noise levels provide a good indication that the detection is operating close to the thermal limit, which is confirmed by test measurements on a graphite sample (see next section).

The differences in the noise levels between $\mathrm{X}$ and $\mathrm{Y}$ might be due to specific details of the interferometer branches (especially the quality of connectors and of the end face of each fiber). We assume that the signal to noise ratio can be further improved by coating the fiber end faces with a metal layer to increase the reflectance of the fiber/air interface (see Sec. II B).

\section{EXPERIMENTAL RESULTS}

For a first testing of the instrument and the complex data acquisition we used a commercial AFM calibration sample with a regular ripple structure of known dimensions. ${ }^{32}$ The employed sample is a glass substrate that has parallel aluminium hills with a period of $278 \pm 1 \mathrm{~nm}$ and a height exceeding $30 \mathrm{~nm}$. The tip was electrochemically etched from a tungsten wire and glued into the Tribolever®. Figures 8(a)-8(c) show topography and friction images that were recorded simultaneously at a constant normal load of $0.85 \mathrm{nN}$. The topography image shows the parallel hill-and-valley structure of the calibration sample. The height of the hills is $33 \mathrm{~nm}$. The friction force images show high frictional forces on top of the aluminium stripes both in $\mathrm{X}$ and $\mathrm{Y}$ direction plus an additional lateral force, where the tip ran against the stripes. A plot of a scan line in the forward and in the backward direction of image 8(b) shows a typical friction loop. When the ridges are aligned perpendicular to the $\mathrm{X}$ direction, the maximum friction force measured in the $\mathrm{X}$ direction is a factor 200 higher than the maximum friction force that is measured in the $\mathrm{Y}$ direction, which shows that the coupling between $\mathrm{X}$ and $\mathrm{Y}$ directions is at most $0.5 \%$. 

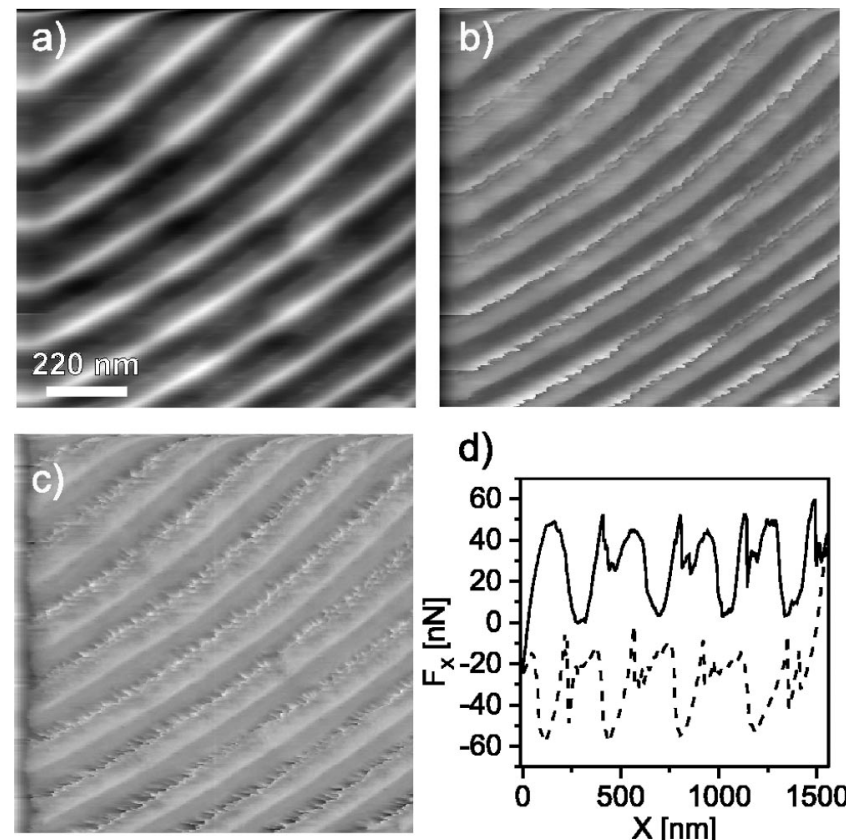

d)

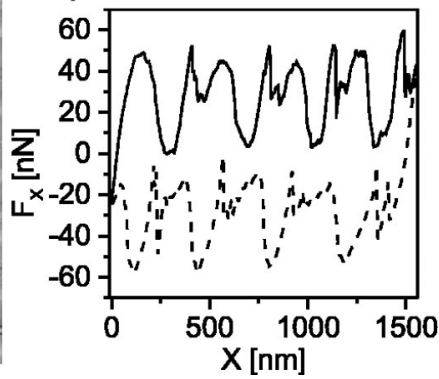

FIG. 8. Simultaneously measured topography and lateral force images of a TDG01 calibration sample. (a) Topography (feedback) image. The gray scale corresponds to $39.8 \mathrm{~nm}$, (b) lateral force image in the $\mathrm{X}$ direction. The gray scale corresponds to $115 \mathrm{nN}$, (c) lateral force image in the $\mathrm{Y}$ direction. The gray scale corresponds to $258 \mathrm{nN}$, (d) friction loop formed by a forward (solid) and backward (dashed) scan line measured in the $\mathrm{X}$ direction. The image size is $1.5 \mu \mathrm{m} \times 1.5 \mu \mathrm{m}$ and the constant normal force is $F_{N}$ $=0.85 \mathrm{nN}$.

As a second test sample we used a highly oriented pyrolytic graphite (HOPG) surface. Figure 9 depicts lateral force maps measured in the forward $\mathrm{X}$ direction and $\mathrm{Y}$ direction $(\mathrm{a}, \mathrm{b})$ and the topography map (c) of two graphite terraces separated by a 0.3-nm-high step. Because of the much higher spring constant in the normal direction the topographic image shows less detail than the lateral force map. As has been reported in several previous articles (e.g. Ref. 33) the lateral force at the step edge is enhanced when the tip is moving step up and also step down.

Figure 10 shows friction forces measured in the $\mathrm{X}$ direction (open circles) and in the $\mathrm{Y}$ direction (open squares) on an atomically flat graphite terrace as a function of the sliding angle. To obtain the friction force in the sliding direction (closed circles) the vector addition of the friction forces in the $\mathrm{X}$ and the $\mathrm{Y}$ direction needs to be computed

$$
F^{F}=F_{X}^{F} \cos \zeta+F_{Y}^{F} \sin \zeta,
$$

where $\zeta$ denotes the angle between the $\mathrm{X}$ direction of the Tribolever and the sliding direction of the tip. Whereas the measured friction force in the $\mathrm{X}$ and the $\mathrm{Y}$ direction varied strongly with the sliding direction, the friction force in the sliding direction stayed nearly constant as expected from a Tomlinson model calculation. This demonstrates the capability of the microscope to measure friction forces in any sliding direction.

The three-dimensional force sensitivity at the atomic scale is demonstrated in Fig. 11. Panels (a),(b),(d),(e) show forward and backward friction maps with the HOPG sample in $\mathrm{X}$ and $\mathrm{Y}$ direction of a 3-nm $\times 3$-nm-wide area. The mea-
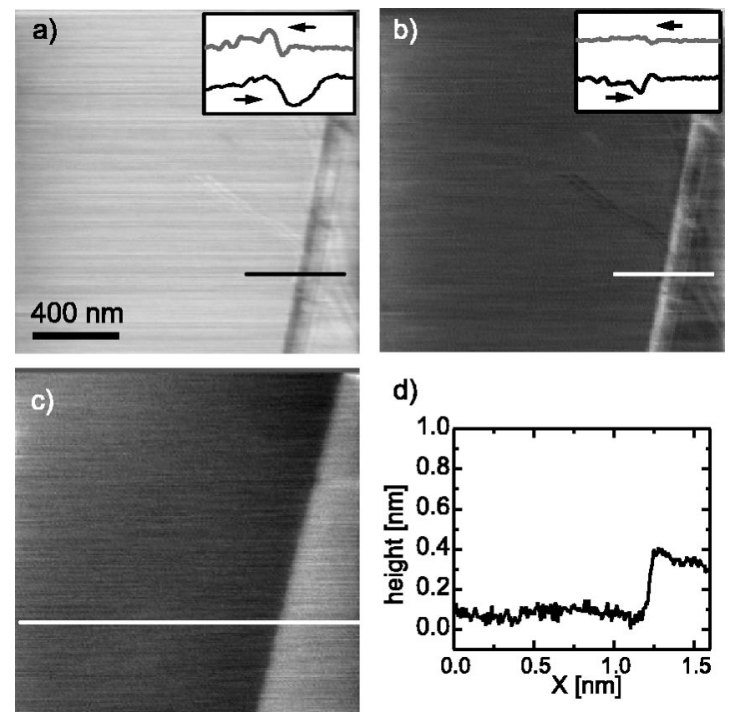

FIG. 9. Correlation between lateral force and topography images of a step running across a HOPG sample. (a) Lateral force image in the forward $\mathrm{X}$ direction. (b) Lateral force image in the forward Y direction. The insets in (a) and (b) show line scans in the forward (black) and in the backward direction (gray), as indicated by a line in both images. The lateral force at the step is enhanced, both in the upward and the downward direction. Note also that the force response of the step is different in the $\mathrm{X}$ and the $\mathrm{Y}$ direction. The lateral force scale of the insets corresponds to $300 \mathrm{nN}$. (c) Simultaneously measured topography image, (d) line scan of the topography image.

surement was performed in a scan direction, which was not aligned along either the $\mathrm{X}$ or the $\mathrm{Y}$ direction of the Tribolever, and atomic scale variations in the friction force could be observed in two directions. The friction loops show a "sawtooth-like" signal for the $\mathrm{X}$ direction [Fig. 11(c)] and a "square-wave"-like signal for the Y direction [Fig. 11(f)]. From these signals it can be deduced that the tip follows a "zig-zag" trajectory on the graphite lattice. ${ }^{34}$ It is important

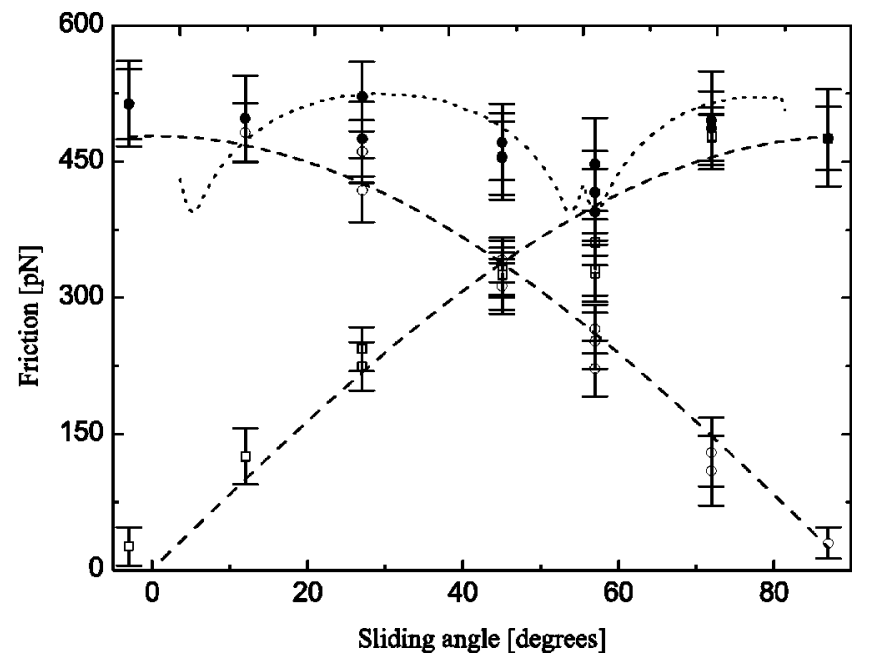

FIG. 10. Friction force on an atomically flat HOPG surface as function of the sliding angle. The open circles denote the friction $F_{X}^{F}$ measured in the $\mathrm{X}$ direction of the Tribolever, the open squares the friction $F_{Y}^{F}$ in the $Y$ direction. The closed circles show the friction $F^{F}$ in the sliding direction calculated from these components, according to Eq. (5). The dashed lines are a sine and a cosine fit to the friction components in the $\mathrm{X}$ and the $\mathrm{Y}$ direction. The dotted line shows calculated friction forces, obtained using a Tomlinson model. 

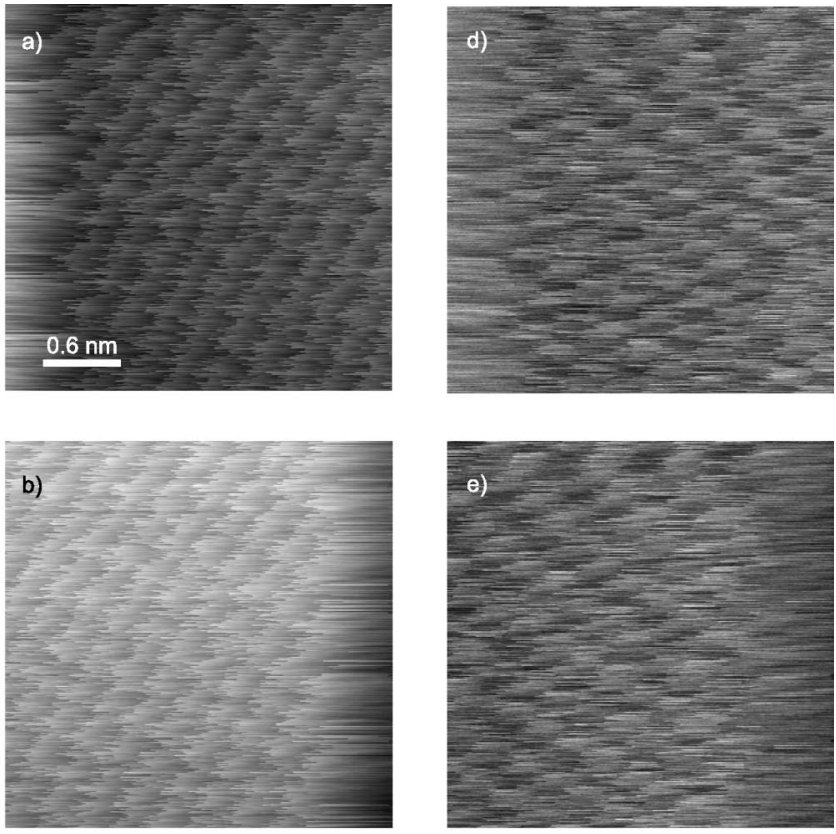

c)

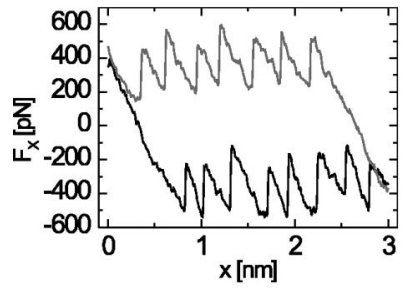

f)

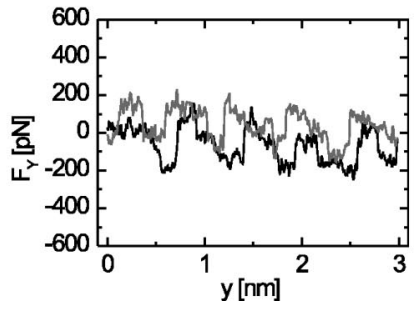

FIG. 11. Lateral force maps for a $\mathrm{W}$ tip on HOPG moving in the $\mathrm{X}$ direction. Lateral forces in the $\mathrm{X}$ direction (a) forward scan. The gray scale corresponds to $1.4 \mathrm{nN}$, (b) backward scan. Gray scale: $1.5 \mathrm{nN}$, (c) friction loop. Lateral force map in the perpendicular Y direction: (d) forward scan. Gray scale: $222 \mathrm{pN}$, (e) backward scan. Gray scale: $243 \mathrm{pN}$, (f) friction loop. All images were measured simultaneously with $F_{N}=35.8 \mathrm{nN}$; image size $3 \mathrm{~nm} \times 3 \mathrm{~nm}$.

to note that the lateral forces measured with our microscope are much lower than in previous studies (e.g. Ref. 35). From the noise in the $\mathrm{X}$ and $\mathrm{Y}$ channels during the friction measurements, we estimate that the lateral force resolution (rms) in the measurement is $15 \mathrm{pN}$ in the $\mathrm{X}$ direction and $41 \mathrm{pN}$ in the $\mathrm{Y}$ direction. The topography image (not shown) does not show any structure, although the feedback system had been set for constant normal force. This implies that the topography and friction signals are completely decoupled.

\section{ACKNOWLEDGMENTS}

The authors gratefully acknowledge H. J. Hug from the University of Basel who helped develop and adapt the sample manipulation motors. This work is part of the research program of the "Stichting voor Fundamenteel Onderzoek der Materie (FOM)" and was made possible by financial support from the "Nederlandse Organisatie voor Wetenschappelijk Onderzoek (NWO).”

${ }^{1}$ D. Dowson, History of Tribology, 2nd ed. (Longman, London, 1998). ${ }^{2}$ I. L. Singer, R. N. Bolster, J. Wegand, S. Fayeulle, and B. C. Stupp, Appl. Phys. Lett. 57, 995 (1990).
${ }^{3}$ U. D. Schwartz, O. Zwörner, P. Köster, and R. Wiesendanger, in Micro/ Nanotribology and Its Applications NATO ASI Series E Vol. 330, edited by B. Bhushan (Kluwer, Dordrecht, 1997), p. 233.

${ }^{4} \mathrm{~J}$. Greenwood, in Fundamentals of Friction: Macroscopic and Microscopic Processes, NATO ASI Series E Vol. 220, edited by I. L. Singer and H. M. Pollock (Kluwer, Dordrecht, 1992), pp. 37-56.

${ }^{5}$ B. Bhushan, J. Israelachvili, and U. Landman, Nature (London) 374, 607 (1995).

${ }^{6}$ R. W. Carpick, N. Agraï, D. Ogletree, and M. Salmeron, J. Vac. Sci. Technol. B 14, 1289 (1995).

${ }^{7}$ P.-E. Mazaran and J.-L. Loubet, Tribol. Lett. 3, 125 (1997).

${ }^{8}$ D. F. Ogletree, R. W. Carpick, and M. Salmeron, Rev. Sci. Instrum. 67, 3298 (1996).

${ }^{9}$ U. D. Schwarz, P. Köster, and R. Wiesendanger, Rev. Sci. Instrum. 67, 2560 (1996).

${ }^{10}$ T.-Y. Fu, Y.-R. Tzeng, and T. T. Tsong, Surf. Sci. 366, L691 (1996).

${ }^{11}$ T. Zijlstra, J. A. Heimberg, E. van der Drift, D. Glastra van Loon, M. Dienwiebel, L. E. M. de Groot, and J. W. M. Frenken, Sens. Actuators, A 84, 18 (2000).

${ }^{12}$ ANSYS $\odot$, finite element analysis software, Ansys, Inc., Canonsburg, PA.

${ }^{13}$ U. Rabe, K. Janser, and W. Arnold, Rev. Sci. Instrum. 67, 3281 (1996).

${ }^{14}$ G. J. Germann, S. R. Cohen, G. Neubauer, G. M. McClelland, and H. Seki, J. Appl. Phys. 73, 163 (1993).

${ }^{15}$ A. Moser, H.-J. Hug, Th. Jung, U. D. Schwarz, and H.-J. Güntherodt, Meas. Sci. Technol. 4, 769 (1993).

${ }^{16}$ D. Rugar, H. J. Mamin, and P. Guethner, Appl. Phys. Lett. 55, 2588 (1989).

${ }^{17}$ Components of the interferometer are: Laser diode: Sony, gain-guided SLD $201 \mathrm{~V}-3, \lambda=780 \mathrm{~nm}, \mathrm{~S} / \mathrm{N}=80 \mathrm{~dB}$ at maximum $2.5 \mathrm{~mW}$ output coupled directly to Faraday isolator and fiber pigtail with an angled polished FC connector (FC/APC) at the output end. Current source: ILX Lightwave, battery controlled LD-2000, <800 nA rms noise, 5 ppm long term (1-20 h) stability. Thermal electric cooler, TEC TC-5100, and high power heat sink from ILX Lightwave, Bozeman, MT. Glass fibers and couplers: Wave Optics, Inc., Hillsboro, OR single-mode, mode field diameter $5.5 \mu \mathrm{m}$, cladding diameter $125 \mu \mathrm{m}$, numerical aperture 0.11 , terminated with FC/APC connectors

${ }^{18}$ Methods developed for making sharp near-field scanning microscopy tips cannot be used in our application because they stretch the core and decrease its diameter as it is melted. This would introduce spurious backreflections into our interferometer.

${ }^{19}$ Wave Optics, Inc., Mountain View, CA.

${ }^{20}$ D. R. Turner, US Patent No. 4,469,554 (1983).

${ }^{21}$ P. Hoffmann, B. Dutoit, and R.-P. Salathé, Ultramicroscopy 61, 165 (1995).

${ }^{22}$ Nanomotor ${ }^{\circledR}$, Klocke Nanotechnik GmbH, 52078 Aachen, Germany.

${ }^{23}$ This idea was first used by Olaf Wolter in the alignment chip (ALIGN) from Nanosensor $\mathrm{GmbH}$ producing repositioning resolutions of $5 \mu \mathrm{m}$. However, the ALIGN systems of ridges and grooves do not form a true kinematic mount. In theory, a trihedral hole is required to form a true kinematic mount. Due to the wet etching characteristics of silicon, a quadrahedral hole is used with very nice results.

${ }^{24}$ Staveley NDT Technologies, Inc., EBL 2 piezo tube, outer diameter 0.25 in., length 0.5 in., wall thickness 0.03 in..

${ }^{25}$ H.-J. Hug, B. Stiefel, P. J. A. van Schendel, A. Moser, S. Martin, and H.-J. Güntherodt, Rev. Sci. Instrum. 70, 3625 (1999).

${ }^{26}$ M. Dienwiebel, G. S. Verhoeven, N. Pradeep, J. W. M. Frenken, J. A. Heimberg, and H. W. Zandbergen, Phys. Rev. Lett. 92, 126101 (2004).

${ }^{27}$ R. Euler, U. Memmert, and U. Hartmann, Rev. Sci. Instrum. 68, 1776 (1997).

${ }^{28}$ Dragonite ${ }^{\circledR}$ glass beads, Jaygo Inc., Union, NJ.

${ }^{29}$ J. P. Cleveland, S. Manne, D. Bocek, and P. K. Hansma, Rev. Sci. Instrum. 64, 403 (1993).

${ }^{30}$ D. Ogletree, R. W. Carpick, and M. Salmeron, Rev. Sci. Instrum. 67, 3298 (1996).

${ }^{31}$ R. D. Grober et al., Rev. Sci. Instrum. 71, 2776 (2000).

${ }^{32}$ TDG01 diffraction grating, NT-MDT Co., Moscow, 103460, Russia.

${ }^{33}$ T. Müller, M. Lohrmann, T. Kässer, O. Marti, J. Mlynek, and G. Krausch, Phys. Rev. Lett. 79, 5066 (1997).

${ }^{34}$ S. Fujisawa, K. Yokoyama, Y. Sugawara, and S. Morita, Phys. Rev. B 58, 4909 (1998).

${ }^{35}$ C. M. Mate, G. M. McClelland, R. Erlandsson, and S. Chiang, Phys. Rev. Lett. 59, 1942 (1987). 